\title{
Deep Reinforcement Learning for 5G Radio Access Network Slicing with Spectrum Coexistence
}

\author{
Yi Shi, Parisa Rahimzadeh, Maice Costa, Tugba Erpek, and Yalin E. Sagduyu
}

\begin{abstract}
The paper presents a reinforcement learning solution to dynamic admission control and resource allocation for 5G radio access network (RAN) slicing requests, when the spectrum is potentially shared between $5 \mathrm{G}$ and an incumbent user such as in the Citizens Broadband Radio Service scenarios. Available communication resources (frequency-time resource blocks and transmit powers) and computational resources (processor power) not used by the incumbent user can be allocated to stochastic arrivals of network slicing requests. Each request arrives with priority (weight), throughput, computational resource, and latency (deadline) requirements. As online algorithms, the greedy and myopic solutions that do not consider heterogeneity of future requests and their arrival process become ineffective for network slicing. Therefore, reinforcement learning solutions $(Q-$ learning and Deep Q-learning) are presented to maximize the network utility in terms of the total weight of granted network slicing requests over a time horizon, subject to communication and computational constraints. Results show that reinforcement learning provides improvements in the $5 \mathrm{G}$ network utility relative to myopic, greedy, random, and first come first served solutions. In particular, deep $Q$-learning reduces the complexity and allows practical implementation as the state-action space grows, and effectively admits/rejects requests when 5G needs to share the spectrum with incumbent users that may dynamically occupy some of the frequency-time blocks. Furthermore, the robustness of deep reinforcement learning is demonstrated in the presence of the misdetection/false alarm errors in detecting the incumbent user's activity.
\end{abstract}

Index Terms-5G, RAN, network slicing, deep reinforcement learning, admission control, resource allocation, spectrum coexistence.

\section{INTRODUCTION}

The demand for high-rate and low-latency communications is ever growing with emerging applications such as virtual/augmented reality and Internet of Things. $5 G \mathrm{com}$ munications has evolved to meet the increasing user demand with its quality of experience (QoE) promises including high throughput and low latency. For cellular communications systems prior to $5 \mathrm{G}$, static allocation of the network resources has been typically considered to support different types of user applications. However, this solution is inefficient and

Yi Shi is with Virginia Tech, Blacksburg, VA, USA; Email: yshi@vt.edu. Parisa Rahimzadeh, Maice Costa, and Yalin E. Sagduyu are with Intelligent Automation, Inc., Rockville, MD, USA; Email: \{prahimzadeh, mcosta, ysagduyu\}@i-a-i.com. Tugba Erpek is with Virginia Tech, Arlington, VA, USA; Email: terpek@vt.edu.

This effort is supported by the U.S. Army Research Office under contract W911NF-21-C0015. The content of the information does not necessarily reflect the position or the policy of the U.S. Government, and no official endorsement should be inferred.

A preliminary version of the material in this paper was partially presented at IEEE International Workshop on Computer Aided Modeling and Design of Communication Links and Networks (CAMAD), 2020 [1].

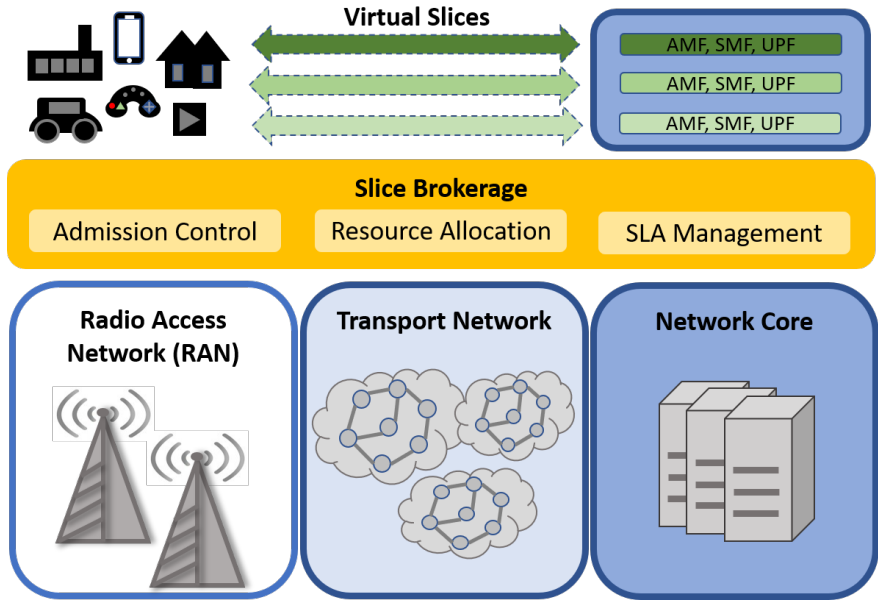

Fig. 1: End-to-end virtual slices in a 5G network architecture.

falls short of meeting the QoE demands in $5 \mathrm{G}$ and beyond communication systems.

5G introduces the Radio Access Network (RAN) slicing capability, where the physical network infrastructure is shared among mobile virtual network operators. The static allocation of resources (such as frequency, power, and computational resources) is replaced by reserving them on the fly with network slicing based on dynamic user demands. The network function virtualization and network slicing paradigms in $5 \mathrm{G}$ have enabled multi-tenancy. Network slicing allows for the partitioning of the whole network (RAN, Transport, and Core) to independent end-to-end parts (see Figure 1), with different applications, resource requests, and Service Level Agreements (SLAs) [2]. Therefore, novel algorithmic solutions are needed for admission control of network slicing requests and resource allocation for admitted network slices. Each network slice consists of multiple virtual network functions, with a resource demand vector [3]. The infrastructure provider (IP) needs new policies on how to assign the limited resources of the network to multiple network slices with specific SLA requests.

Figure 1 shows the scenario of end-to-end virtual slices in a $5 \mathrm{G}$ network architecture. Each virtual network function of a network slice has a resource demand vector [3]. The IP assigns the resources of the physical network like Base Stations (BSs), transmission powers, antennas, and resource blocks (RBs) to heterogeneous network slicing requests over time [4], either in batch service or service upon arrival mode [2].

The use cases to be supported by network slicing are categorized as enhanced Mobile Broadband (eMBB), massive 
machine-type communications (mMTC), and ultra-reliable low-latency communications (urLLC), based on the throughput and latency requirements. The details on resource allocation as part of RAN slicing are not defined yet in the 3GPP standards. To address this gap, the ongoing research has focused on how to allocate the resources as part of RAN slicing [5]-[8]. In practice, the control of RAN slices can be implemented in near-real time RAN Intelligent Controller (RIC) of Open-RAN (O-RAN) to support micro-service-based applications called xApps.

Since spectrum is a scarce resource, in an effort to develop better utilization of spectrum and support the increasing data rate requirements for emerging wireless applications, the Federal Communications Commission (FCC) adopted rules for shared commercial use of the $3550-3700 \mathrm{MHz}$ band which is also known as 3.5-GHz Citizens Broadband Radio Service (CBRS) band [9]. A three-tiered access and authorization framework is developed to accommodate the shared federal and non-federal use of the band. Since $5 \mathrm{G}$ and a radar system (the incumbent user) use these bands, it is required that $5 \mathrm{G}$ does not interfere with the incumbent user. One approach to achieve this spectrum coexistence goal is to continuously sense the spectrum by the Environmental Sensing Capability (ESC) sensors and turn off the $5 \mathrm{G}$ operation once an incumbent signal is detected. However, depending on the radar bandwidth, a naive solution to pause the operation of the $5 \mathrm{G}$ network may be infeasible. A better approach would be not to allocate the corresponding spectrum by the Spectrum Access System (SAS) to the commercial users of $5 \mathrm{G}$ once an incumbent signal is detected at a specific resource block. Taking this spectrum sharing scenario into account, in this paper, we consider the dynamic allocation of spectrum resources to $5 \mathrm{G}$ network slicing requests, in the presence of the incumbent user.

\section{A. Related Work}

In RAN slicing, the complex network dynamics makes the underlying network optimization problem challenging. In [10], the authors considered both network slicing and mobile edge computing technologies, and showed that the resource allocation problem formulated to minimize the interference among different mobile virtual network operators is NP-hard. [4] and [10] considered RAN slicing at the RB level, to minimize interference and improve multi-BS coordination. [11] modeled the resource assignment to multiple requests in a time window with a geometric knapsack problem. [3] proposed centralized and distributed (based on convex optimization problem and auction game) resource allocation algorithms for different slices in a cloud RAN scenario, considering limited amount of resources at data centers. [3] considered two scenarios of slices being/not being honest about their resource requirements and the variable is the amount of resources allocated to each slice (number of users it can serve).

Recently, machine learning was applied to solve the RAN optimization problem as an alternative to the model-based optimization that becomes easily intractable due to the complexity of dynamics involving resources and requests. [12] showed that in-network deep learning is promising for application and device specific identification and traffic classification problems. Deep learning was also used in [13] to manage network load efficiency and network availability.

As data may not be readily available to train complex deep learning structures for resource allocation in response to network slicing requests, a model-free approach such as reinforcement learning (RL) has emerged as a practical solution to learn from the $5 \mathrm{G}$ network environment and update resource allocation decisions for network slicing. For resource allocation as part of network slicing, reinforcement learning was compared to the static and round-robin scheduling methods in [14]. Both bandwidth and computational resources were considered in [2] for network slicing subject to both service upon arrival and batch service modes. In [15], RL-based resource allocation was compared to heuristic, best-effort, and random methods. [16] showed a prototype of network slicing implemented in an end-to-end mobile network system. [17] used reinforcement learning for power-efficient resource allocation in cloud RANs considering multiple transmitters and receivers at the same frequency (rather than $5 \mathrm{G}$ time-frequency blocks). In [18], resource allocation with reinforcement learning was considered by exploiting prediction on communication requests. Reinforcement learning was also used extensively for resource allocation in wireless applications other than network slicing [19]-[21]. [22] considered the unused resources of the network to be added to the resources of each slice, in case they need additional resources (resource reservation). This problem was formulated using Deep RL, where the actions are the changes in assigned resources of each slice. 5G RAN slicing with reinforcement learning was also studied from the security point of view in [23], [24].

Previous work on $5 \mathrm{G}$ network slicing often fails to specify or fully characterize the states, actions, and rewards used in RL. The existing literature also lacks detailed models to describe the available communication and computation resources, in addition to the objectives of a request with respect to latency, throughput (5G rate), priorities, and deadlines. Furthermore, the constraints imposed on resources due to potential spectrum utilization of incumbent signals in the same band have not been considered before.

\section{B. Contributions}

In this paper, we present a deep reinforcement learning model to dynamically allocate resources for $5 \mathrm{G}$ network slicing, as a central admission controller. We consider heterogeneous network slicing request arrivals, modeled as a stochastic process. Arriving requests are directed to the waiting queue, where each request is characterized by the resource requirement and an associated priority. The central controller gathers the waiting requests as well as the network available resources to make the admission control decision for requests waiting in the queue. This admission control problem is formulated as a Markov decision process and solved with RL. In the proposed model, the state is the set of available resources and requests. The state transitions over time depending on 
how these resources are occupied (by granted network slicing requests or the incumbent user) or released (by completed requests). The actions are the admission control decisions on arrived requests. The reward is the network utility measured as the weighted sum of requests satisfied, where weights correspond to relative priorities of these requests.

The motivation of our RL-based solution is that a myopic solution that only considers resources and demands at a given instant cannot be effective in optimizing resources over a time horizon, as current resource allocation will make some resources unavailable for future, thereby coupling the current and future resource allocation problems. As a conventional reinforcement learning algorithm, Q-learning is designed to learn the Q-value, namely the value of an action in a particular state. However, the maintenance of Q-tables becomes computationally infeasible as the number of Q-value evaluations grows with the state-action space. On the other hand, deep reinforcement learning approximates the Q-values by training a deep Q-network (DQN).

We consider dynamic allocation of the RBs, transmit power and computational resources to support downlink communications from a $5 \mathrm{G}$ base station, gNodeB, to user equipments (UEs), in a spectrum sharing scenario. Each network slicing request from a UE is associated with priority, throughput, CPU usage and latency (deadline) requirement, and needs to be served for a specific duration (without interruption from any other user). These requests compete for frequencytime resource blocks, transmit powers of the gNodeB, and computational resources spent to support their applications.

We show that our proposed solution (developed as Qlearning and deep Q-learning) successfully admits network slicing requests over a time horizon and provides major gains in network utility compared to myopic, greedy, random, and first come first served (FCFS) admission control algorithms. As the number of UEs increases or the priorities of network slicing requests change over time, we show that our RL-based solution successfully adapts to dynamic user demands. We further show that our reinforcement learning solution can be effectively used to dynamically allocate spectrum resources in response to incumbent users coexisting with $5 G$ users.

We show that the proposed solution adapts to spectrum sharing dynamics successfully and allocates resources for $5 \mathrm{G}$ RAN slicing effectively in response to dynamic spectrum utilization of incumbent users. It is not a trivial task to reallocate the spectrum resources to network slices in $5 \mathrm{G}$ in response to incumbent user dynamics that change the availability of frequency-time blocks over time. As reinforcement learning takes the time-horizon into account instead of pursuing a myopic objective, it emerges as a viable solution to reconfigure network resources in order to support spectrum coexistence applications for $5 \mathrm{G}$. We show that the proposed solution adapts to spectrum sharing dynamics successfully and allocates resources for 5G RAN slicing effectively in response to dynamic spectrum utilization of incumbent users. We consider the potential errors in detecting the presence of the incumbent user (misdetection and false alarm errors) as well, and demonstrate the robustness of our RL-based solution in the presence of spectrum sensing (signal detection) errors.

The rest of the paper is organized as follows. Section II describes the resources and the network slicing requests, defines the optimization problem for dynamic resource allocation, and presents extensions for more general scenarios. Section III develops reinforcement learning algorithm for the network slicing problem. Section IV provides the performance evaluation results. Section V concludes this paper.

\section{5G RAN Resources AND Network SLICING REQUESTS}

\section{A. System Model}

The system model is shown in Figure 2. Suppose that there is one gNodeB and there are $N$ UEs in a $5 \mathrm{G}$ network. Each UE needs to connect to the gNodeB to have network access for its applications. 5G network supports three types of traffic: eMBB, URLLC, and mMTC. Consequently, UEs may request downlink communication service (from the gNodeB to the UEs) with dynamic QoE levels regarding different throughput, CPU usage and latency (deadline) requirements, and different priorities (relative importance).

Requests are handled by the gNodeB and then appropriate network slices with corresponding resource blocks are assigned to requests. If a request is not answered yet, it stays in a queue until its deadline (time limit from the request arrival until the service starts) expires. The objective of such assignments is to maximize the weighted number of supported requests or the total provided services, where weights represent priorities of these requests. As network resources, we consider bandwidth, communication (transmit) power, and CPU usage. At time $t$, there are a set of active requests $A(t)$ that includes either requests that have just arrived or requests that are in the waiting list (i.e., requests that are not satisfied yet and their deadlines have not expired). The QoE can be measured in different forms, for example throughput or CPU usage. The CPU usage requirement of UE $i$ for its network slicing request $j$ is given by

$$
P_{i j}^{C} \geq p_{i j}^{C}, \quad(i, j) \in A(t),
$$

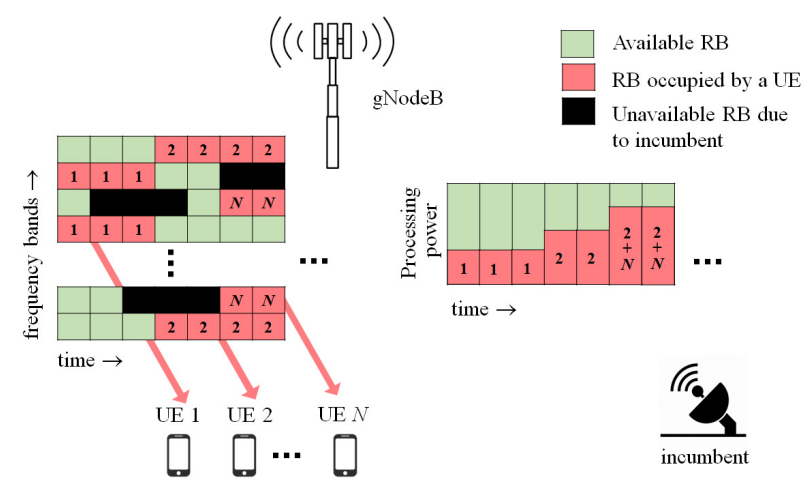

Fig. 2: System model for RAN resource allocation with network slicing. 
where $P_{i j}^{C}$ is the assigned computational resource (measured by CPU usage) and $p_{i j}^{C}$ is the minimum required resource. The throughput requirement of UE $i$ for its request $j$ is given by

$$
D_{i j} \geq d_{i j}, \quad(i, j) \in A(t),
$$

where $D_{i j}$ is the achieved data rate and $d_{i j}$ is the minimum required rate. $D_{i j}$ is determined by the assigned bandwidth $F_{i j}$, the assigned transmit power $P_{i j}^{T}$ (for downlink traffic) at the gNodeB, the modulation coding scheme used for communications between the gNodeB and UE $i$, and channel effects including interference and path loss. Note that each antenna of the gNodeB serves a different user through spatial multiplexing. For 5G NR, the approximate data rate (in bps) for a given number of aggregated carriers in a band or band combination is computed as [25]:

$$
r=\sum_{k=1}^{K}\left(v_{\mathrm{Layers}}^{(k)} Q_{m}^{(k)} f^{(k)} R_{\max } \frac{N_{\mathrm{PRB}}^{B(k), \mu} 12}{T_{s}^{\mu}}\left(1-O^{(k)}\right)\right),
$$

where $K$ is the number of aggregated component carriers (CCs) in a band or band combination, and $R_{\max }=948 / 1024$. For the $k$ th CC, $v_{\text {Layers }}^{(k)}$ is the maximum number of supported layers, $Q_{m}^{(k)}$ is the maximum supported modulation order, $f^{(k)}$ is the scaling factor that can take values $1,0.8,0.75$ or $0.4, \mu$ is the numerology defined in [26], $T_{s}^{\mu}$ is the average OFDM symbol duration in a subframe, where $T_{s}^{\mu}=\frac{10^{-3}}{14 \cdot 2^{\mu}}$ for normal cyclic prefix, $N_{\mathrm{PRB}}^{B(k), \mu}$ is the maximum resource block allocation in the UE-supported maximum bandwidth $B^{(k)}$ in the given band (or combination), and $O^{(k)}$ is the overhead (equal to 0.08 for the uplink in frequency range 1). Assuming a single antenna UE with QPSK modulation, $60 \mathrm{kHz}$ subcarrier spacing and $10 \mathrm{MHz}$ bandwidth, (3) becomes $r=c \times K$, where constant $c$ is approximately $12.59 \times 10^{6}$.

The achieved data rate $r$ is further reduced by the corresponding bit error rate (BER) assuming that low-density parity-check (LDPC) coding is used as forward error correction. To calculate the BER at different SNR levels, we simulated the performance of QPSK signal in additive white Gaussian noise (AWGN) channel with LDPC coding (see Figure 3). We ran the simulation $5 \times 10^{6}$ times (with different noise values). The BER value is 0 for SNR values higher than $-1 \mathrm{~dB}$. The data rate $r$ is scaled based on the BER performance at a given SNR. Thus, when $K_{i j}$ is the number of aggregated CCs and $B E R_{i j}$ is the BER of UE $i$ for its request $j$, (2) becomes

$$
c K_{i j}\left(1-B E R_{i j}\right) \geq d_{i j}, \quad(i, j) \in A(t) .
$$

\section{B. Optimization Problem for Dynamic Resource Allocation to Network Slices}

Denote $x_{i j}(t)$ as the binary indicator on whether UE $i$ 's request $j$ is satisfied at time $t$. The constraints of resource

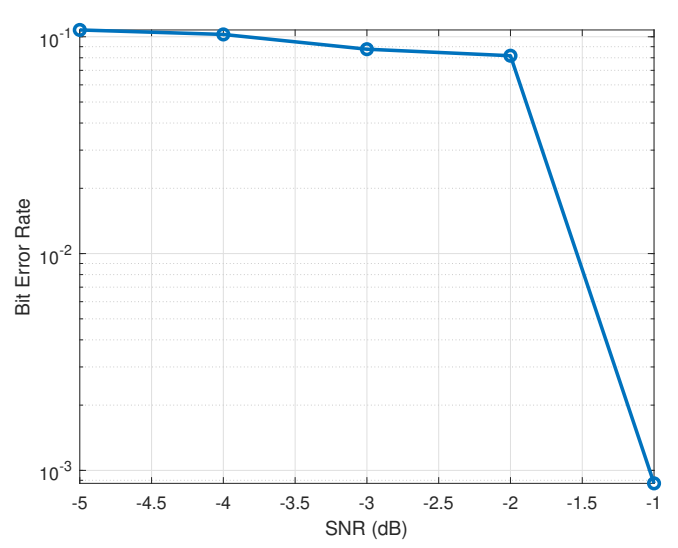

Fig. 3: BER vs. SNR for QPSK signal with LDPC coding in AWGN channel.

assignments to network slices are given as follows.

$$
\begin{aligned}
& \sum_{(i, j) \in A(t)} F_{i j} x_{i j}(t) \leq F(t), \\
& \sum_{(i, j) \in A(t)} P_{i j}^{C} x_{i j}(t) \leq P^{C}(t), \\
& \sum_{(i, j) \in A(t)} P_{i j}^{T} x_{i j}(t) \leq P^{T}(t),
\end{aligned}
$$

where $F(t), P^{C}(t)$ and $P^{T}(t)$ are available communication (frequency-time blocks), computational and transmit power resources, respectively, at the gNodeB at time $t$. Some of the gNodeB's resources may have been already assigned to some requests, which are not terminated yet, and thus some resources may not be available.

The myopic objective that optimizes the resource allocation at time $t$ only is to select $F_{i j}(t), P_{i j}^{C}(t)$ and $P_{i j}^{T}(t)$ for

$$
\max \sum_{(i, j) \in A(t)} w_{i j} x_{i j}(t),
$$

subject to (1), (4)-(7), where $w_{i j}$ is the weight for UE $i$ 's request $j$ to reflect its priority. In (8), the myopic objective function is the reward of network slicing allocation at time $t$.

Next, we consider the optimization problem for a time horizon. The resources are updated from time $t-1$ to time $t$ as follows.

$$
\begin{aligned}
F(t) & =F(t-1)+F_{r}(t-1)-F_{a}(t-1), \\
P^{C}(t) & =P^{C}(t-1)+P_{r}^{C}(t-1)-P_{a}^{C}(t-1), \\
P^{T}(t) & =P^{T}(t-1)+P_{r}^{T}(t-1)-P_{a}^{T}(t-1),
\end{aligned}
$$

where $F_{r}(t-1), P_{r}^{C}(t-1)$ and $P_{r}^{T}(t-1)$ are released resources at time $t-1$ on frequency, CPU usage, and transmit power, respectively, and $F_{a}(t), P_{a}^{C}(t)$ and $P_{a}^{T}(t)$ are allocated resources at time $t$ on frequency, CPU usage and transmit power, respectively. Each request has a lifetime $l_{i j}$ and if it is satisfied at time $t$ (namely, the service starts at time $t$ ), this request will end at time $t+l_{i j}$. Denote $R(t)$ as the set of requests ending (completed or expired) at time $t$. The released 
and allocated (communications and computational) resources at time $t$ are given by

$$
\begin{aligned}
F_{r}(t) & =\sum_{(i, j) \in R(t)} F_{i j}, \\
P_{r}^{C}(t) & =\sum_{(i, j) \in R(t)} P_{i j}^{C}, \\
P_{r}^{T}(t) & =\sum_{(i, j) \in R(t)} P_{i j}^{T},
\end{aligned}
$$

and

$$
\begin{aligned}
F_{a}(t) & =\sum_{(i, j) \in A(t)} F_{i j}, \\
P_{a}^{C}(t) & =\sum_{(i, j) \in A(t)} P_{i j}^{C}, \\
P_{a}^{T}(t) & =\sum_{(i, j) \in A(t)} P_{i j}^{T} .
\end{aligned}
$$

Then, the optimization problem changes to

$$
\max \sum_{t} \sum_{(i, j) \in A(t)} w_{i j} x_{i j}(t),
$$

subject to (1), (4)-(7), (9)-(17). In (18), the objective function is the reward of network slicing allocation over a time horizon. Under an unrealistic assumption that the gNodeB knows all future requests in advance, this problem can be solved offline. Future requests can be predicted by a deep neural network [27]. Then, resources can be allocated using reinforcement learning [18]. In this paper, we solve this problem by reinforcement learning without any knowledge on future requests, as we will discuss in Section III.

\section{Extensions}

We extend this formulation to dynamic spectrum sharing of $5 \mathrm{G}$ with an incumbent legacy communication system. One example is the CBRS band [9]. The FCC authorized the use of the CBRS band for wireless service provider commercialization. In this dynamic spectrum access setting, radar is the incumbent access user (primary user) and $5 \mathrm{G}$ is the priority access user (secondary user). The ESC sensors need to detect the radar signal with spectrum sensing (potentially using statistical [28] and machine learning [29] techniques). When the radar signal is detected, the SAS needs to reconfigure the $5 \mathrm{G}$ resource allocation to assign network slices to frequency bands that are not yet occupied by the radar. In our formulation, the available resources $A(t)$ are updated by possibly removing some frequency blocks temporarily due to radar signal occupancy. In addition, we consider the possible misdetection and false alarm errors in detecting the incumbent activity. We assume that the ESC fails in detecting an incumbent-occupied RB with probability of misdetection, $p_{M}$, and flags an available $\mathrm{RB}$ as an occupied one with probability of false alarm, $p_{F}$.

This formulation can be extended to multiple gNodeBs, each with its own transmit power and processing power. One naive approach is to allocate resources independently. However, frequency resource blocks should be jointly allocated due to potential interference relationships. In addition to reinforcement learning considered per gNodeB, the following preprocessing and post-processing stages need to be performed:

1) Pre-processing. A resource block is unavailable if (i) it was already allocated to some earlier requests and (ii) it was used by neighboring gNodeBs. Each gNodeB can sense the spectrum and try to identify the resource blocks that are used by neighboring gNodeBs. Alternatively, a gNodeB can also obtain such information in the postprocessing stage.

2) Post-processing. Neighboring gNodeBs may plan to assign the same resource blocks in their reinforcement learning algorithms. To avoid interference among these assignments, a central controller can be used to resolve such conflicts (by removing some assignments) and broadcast the remaining assignments. A distributed scheme is also possible by letting each gNodeB locally broadcast its planned assignments and compare them with planned assignments from neighboring gNodeBs to decide whether a planned assignment should be kept or not.

\section{REINFORCEMENT LEARNING ALgORITHM FOR 5G RAN SLICING}

This section describes the reinforcement learning solution used to optimize our $5 \mathrm{G}$ slice admission control policy. In the beginning of each time slot, we consider all requests in the queue. These requests include both newly incoming requests in the previous time slot and older requests with remaining deadline. It is important to avoid accepting requests which need RBs that are previously occupied by an incumbent user. This process is subject to misdetection errors (with probability $p_{M}$ ) and false alarm errors (with probability $p_{F}$ ) in detecting the incumbent user activity.

Reinforcement learning models the decision-making in the interaction of an agent taking actions in a changing environment over different time slots. The state consists of (i) network status part and (ii) request-specific part.

(i) The network status part of the state represents the occupied resources of the network, including the status of occupied RBs by an incumbent user, the status of occupied RBs by requests in service, amount of used computational resources, and amount of used communication power. Note that the status of occupied RBs by an incumbent, in the state vector, is the status from the controller point of view, which entails possible misdetection and false alarm errors.

(ii) The request-specific part represents the characteristics of the considered request for admission. This information includes the required number of RBs and communication power determined by the throughput requirement, the required amount of processor usage, weight, and duration of the considered request. 
The set of actions are the admission control decisions of accepting or rejecting the considered request. The rejected request will stay in the queue until it will be accepted at a later time slot or dropped when it is overdue.

The immediate reward of accepting a request in a time slot depends on the weight of that request. If the presence of an incumbent user is not detected correctly, i.e., if a misdetection occurs, the request, using at least one overlapping RB, will be dropped. Then, losing this request in service will be reflected in the reward of that time slot.

The goal is to find the optimal policy which maximizes the average cumulative discounted reward. We use Q-learning and deep Q-learning algorithms as the model-free reinforcement learning algorithms to learn the optimal policy that determines which action (admission control) to take under a given state (available resources and request) for the gNodeB. The gNodeB as a central controller applies these algorithms to compute the function $Q: S \times A \rightarrow \mathbb{R}$ to evaluate the quality of action $a \in A$ producing reward $R$ at state $s \in S$.

The optimal Q-function satisfies the Bellman equation

$$
Q^{*}(s, a)=\mathbb{E}_{r, s^{\prime}}\left[r+\gamma \max _{a^{\prime}} Q^{*}\left(s^{\prime}, a^{\prime}\right) \mid s, a\right],
$$

where the expectation is over both the distribution of immediate rewards $r$ and possible next states $s^{\prime}$. The Q-learning algorithm solves the Bellman equation by iteratively updating the Q-function, while deep Q-learning solution uses deep neural networks to approximate the Q-function. Next, we describe the Q-learning and deep Q-learning solutions in Sections III-A and III-B, respectively.

\section{A. Q-Learning Algorithm}

Using the Q-learning algorithm, the gNodeB maintains $Q$ as the Q-table. At each time $t$, the gNodeB selects an action $a_{t}$, observes a reward $r_{t}$, and transitions from the current state $s_{t}$ to a new state $s_{t+1}$ (this transition depends on current state $s_{t}$ and action $a_{t}$ ), and updates $Q$.

Starting $Q$ as a random matrix and using the weighted average of the old value and the new information, Q-learning performs the value iteration update for $Q$ as follows:

$$
\begin{aligned}
Q\left(s_{t}, a_{t}\right) \leftarrow & Q\left(s_{t}, a_{t}\right) \\
& +\alpha \cdot\left(r_{t}+\gamma \cdot \max _{a} Q\left(s_{t+1}, a\right)-Q\left(s_{t}, a_{t}\right)\right),
\end{aligned}
$$

where $\alpha$ is the learning rate $(0<\alpha \leq 1)$ and $\gamma$ is the discount factor $(0 \leq \gamma \leq 1)$ for rewards over time. In (20), $\max _{a} Q\left(s_{t+1}, a\right)$ refers to the estimate of the optimal future value of $Q$.

In dynamic resource allocation to network slices, the reward at time $t$ is $w_{i j}$ if $\mathrm{UE} i$ 's request $j$ is satisfied at time $t$, i.e., $x_{i j}(t)=1$. An action of the gNodeB at time $t$ corresponds to the assignment of resources to a request at time $t$. Note that multiple actions can be taken at the same time instance. The transition of the state at time $t$ is driven by blocking resources for requests that are granted at time $t$ and releasing resources after the lifetimes of active services expire at time $t$. In particular, the corresponding state transitions are given by (9)-(17).

\section{B. Deep Q-learning Algorithm}

As the state-action space increases in size, the Q-learning algorithm becomes inefficient and computationally expensive, as it is necessary to keep a large Q-table for state-action pairs. To bridge the gap between high-dimensional inputs and actions, the work in [30] combines reinforcement learning and deep neural networks, and enhances the conventional Qlearning algorithm by approximating the optimal action-value function with a non-linear deep neural network. The DQN maps history-action pairs to a parameterized approximate value function $Q\left(s, a ; \theta_{i}\right)$, where the parameter $\theta_{i}$ sets the weights of the neural network at the $i$-th iteration. We follow a similar approach, using the state representation as an input to the DQN, and a separate output for each possible action, the estimated Q-values associated to the input state.

Approximating the value function with nonlinear estimators as a neural network is known to cause instability in RL, and the solution in [30] addressed this issue by adopting two techniques, namely a separate target DQN, and the use of a biologically inspired mechanism termed experience replay. In experience replay, past experiences $e_{t}=\left(s_{t}, a_{t}, s_{t+1}, r_{t}\right)$ are stored in the replay memory. The training is performed using samples drawn uniformly at random from the replay memory, reducing the correlation found in sequential observations and increasing efficiency when training the network, as one step of experience may be reused in many weight updates. Experience replay is used to learn off-policy while reducing oscillations or divergence in the parameters.

The DQN is trained by adjusting the parameters $\theta_{i}$ at each iteration to minimize the mean-squared error in the Bellman equation, while replacing the optimal target values with approximated target values $y_{i}=r+\gamma \max _{a^{\prime}} Q\left(s^{\prime}, a^{\prime} ; \theta_{i}^{-}\right)$, using parameters $\theta_{i}^{-}$from a previous iteration. The Q-learning update at each iteration aims to minimize the loss given by

$$
L=\left(y_{i}-Q\left(s, a ; \theta_{i}\right)\right)^{2},
$$

where the parameters used to compute the target value at the $i$ th iteration are periodically updated with the DQN parameters and held constant in-between updates. [30] uses a separate network to determine the target values $y_{i}$ in each iteration and replaces the target network with the DQN every $C$ iterations, improving stability in comparison to online Q-learning.

The overview of the proposed admission control solutions based on deep Q-learning is illustrated in Figure 4. The current state $s$ used as the input includes the current availability of network resources and the features associated to the requests, while the two outputs are $Q\left(s\right.$, 'accept'; $\left.\theta_{i}\right)$ and $Q\left(s\right.$, 'reject'; $\left.\theta_{i}\right)$. We normalize the input to expedite the training of the DQN. The optimal admission control policy is learned by interacting with the environment based on the $\epsilon$-greedy method, following the greedy policy $a=$ $\operatorname{argmax}_{a} Q\left(s, a ; \theta_{i}\right)$ with probability $1-\epsilon$ and selecting a random action with probability $\epsilon$. 


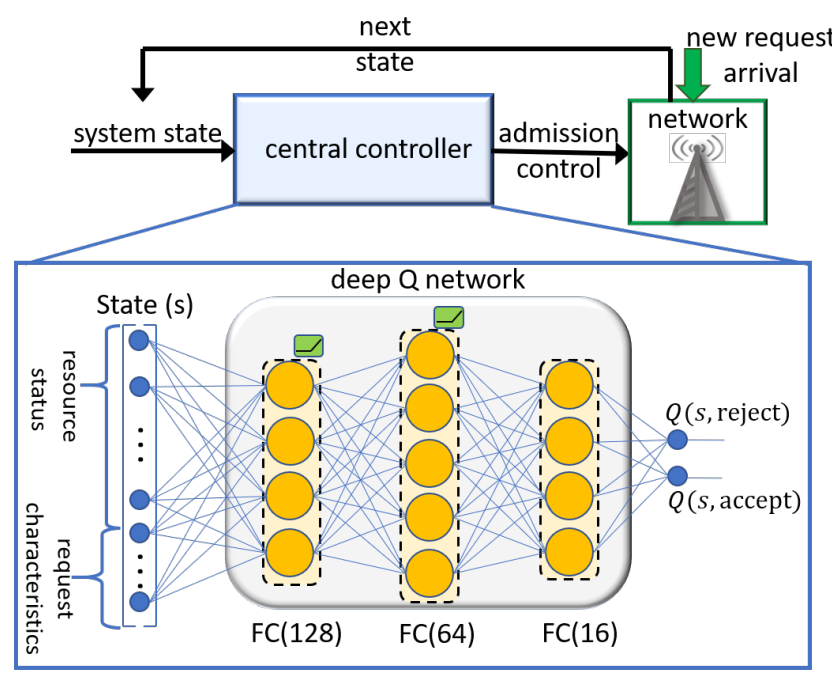

Fig. 4: The overview of the proposed admission control solutions based on deep Q-learning.

\section{Performance Evaluation}

Suppose the gNodeB receives requests possibly from three UEs. Later, we extend this setting to more UEs. For each UE, requests arrive with rate of 0.5 per slot. Here, a slot corresponds to each time block that is $0.23 \mathrm{~ms}$ long with $60 \mathrm{kHz}$ subcarrier spacing. The CPU is measured from $0 \%$ to $100 \%$ by $2 \%$ increments (with total of 50 levels). For each request, weight is a random integer in [1,5], lifetime is assigned randomly in [1,10] slots, and deadline is assigned randomly in $[1,20]$ slots. Transmit power is set from 5 levels and the maximum received SNR is selected randomly in $[1.5,3]$. The total bandwidth is $10 \mathrm{MHz}$ and is split into 11 bands. The same scenario over 1000 time slots is run to test different algorithms, namely Q-learning, random, FCFS, and myopic algorithms. For Q-learning, we set discount factor as $\gamma=0.95$ and learning rate as $\alpha=0.1$.

TABLE I: Performance comparison of Q-learning and baseline algorithms.

\begin{tabular}{c|c|c} 
Algorithm & Network utility & Ratio of improvement \\
\hline \hline Q-learning & 1807 & - \\
\hline Myopic & 1456 & $24.11 \%$ \\
\hline FCFS & 1416 & $27.61 \%$ \\
\hline Random & 1334 & $35.46 \%$
\end{tabular}

The other three algorithms that we use as benchmarks are described below.

1) Random algorithm: Available resources are allocated to uniformly randomly selected network slice requests.

2) FCFS algorithm: Available resources are allocated to network slice requests based on the arrival times of requests, i.e., at any given time, the oldest network slice request is answered first provided that the available resources are sufficient to grant this request.

3) Myopic algorithm: Available resources are allocated to maximize the current utility only by solving the optimization problem (8).

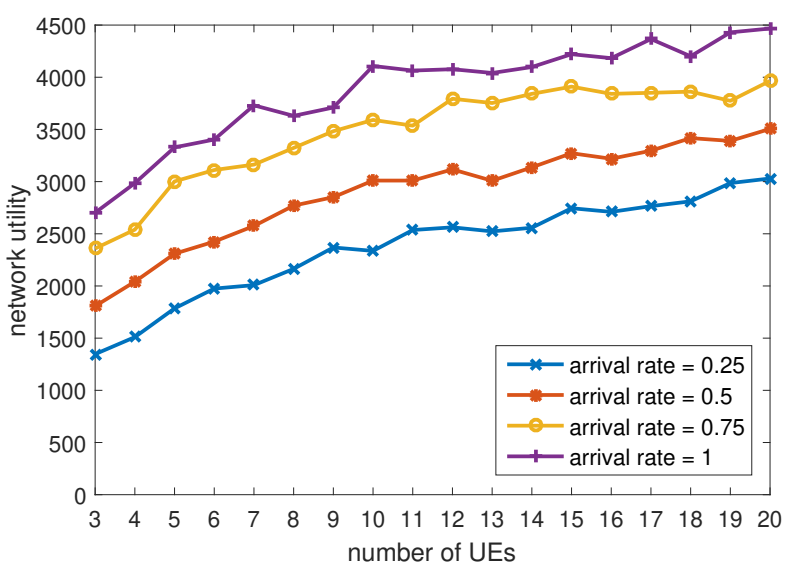

Fig. 5: Network utility vs. number of UEs.

Performance comparison of Q-learning and baseline algorithms is given in Table I that shows the network utility achieved by each algorithm and the ratio of network utility improvement by Q-learning relative to other algorithms. Results show that Q-learning achieves much larger utility (total weights of satisfied requests) than other algorithms. On the other hand, myopic algorithm chooses the current best decision without consideration on future and performs better than FCFS and random resource allocations but falls far behind Q-learning. We run the best two algorithms (Q-learning and myopic) multiple times. The range of achieved utility is measured as $[1731,1831]$ for Q-learning and $[1359,1466]$ for myopic algorithm. These results indicate that Q-learning achieves better utility than myopic algorithm for the entire range of results.

Next, we increase the number of UEs that send network slicing requests to the gNodeB and change the arrival rate at UEs. Figure 5 shows how the network utility scales with the number of UEs when Q-learning is used. Note that the increase of network utility with the increasing number of UEs declines as the remaining resources become less.

In optimization problem (18), weight $w_{i j}$ assigns priority to request $j$ of UE $i$. Next, we evaluate the effect of weights on optimization results. For that purpose, we fix the weight of all requests of one UE (out of three UEs). The first case is $[1,1,1]$, i.e., all requests have a weight 1 . The second case is [1,3,5], i.e., all UE 1's requests have weight 1 , all UE 2's requests have weight 3 , and all UE 3 's requests have weight 5 . The third case is $[5,3,1]$. i.e., all UE 1's requests have weight 5 , all UE 2's requests have weight 3, and all UE 3's requests have weight 1 . We show the number of served requests for each UE in Table II. Results indicate that if a UE's weight is increased and it is larger than others, the number of served requests for this UE increases relative other UEs. On the other hand, the actual values of weights do not play as big a role as the order of weights in terms of the effect on the network utility, i.e., weights $[1,2,3]$ achieve the same result as weights $[1,3,5]$.

We further consider the 5G-radar spectrum coexistence 
TABLE II: The impact of weight on the number of served requests.

\begin{tabular}{c|c} 
Weight of UEs & Number of served requests \\
\hline \hline$[1,1,1]$ & {$[173,139,169]$} \\
\hline$[1,3,5]$ & {$[81,117,278]$} \\
\hline$[5,3,1]$ & {$[274,129,68]$}
\end{tabular}

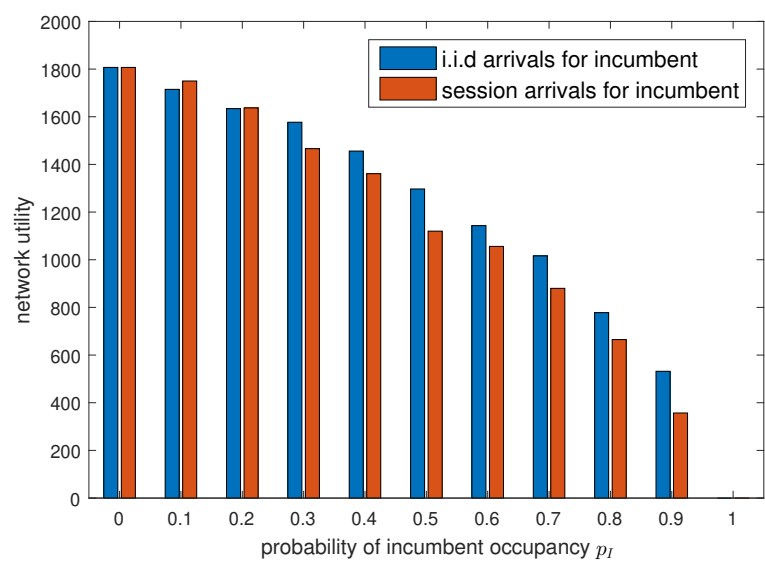

Fig. 6: Network utility when $5 \mathrm{G}$ shares the spectrum with an incumbent user.

scenario, where the radar signals possibly occupy multiple frequency blocks over time. We consider two types of arrival patterns for radar signals. The first pattern corresponds to independent identically distributed (i.i.d.) arrivals and radar signal appears at any time (slot) with probability $p_{I}$. The second pattern corresponds to session (bursty) arrivals and the lifetime of sessions is selected uniformly randomly from $[10,50]$ slots. Then, the arrival of sessions is adjusted to obtain $p_{I}$ as the probability of incumbent occupancy. First, we assume that the radar signal is reliably detected. We will relax this rather unrealistic assumption later and introduce misdetection and false alarm errors to signal detection.

In Figure 6, we show the network utility achieved by Q-

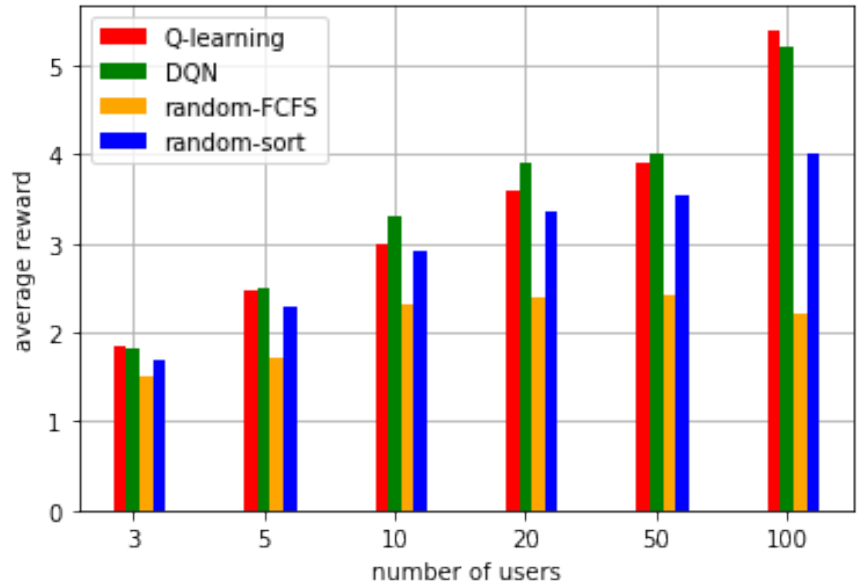

Fig. 7: The comparison of Q-learning and deep Q-learning (based on DQN) algorithms in terms of the average reward of an episode versus the number of users.

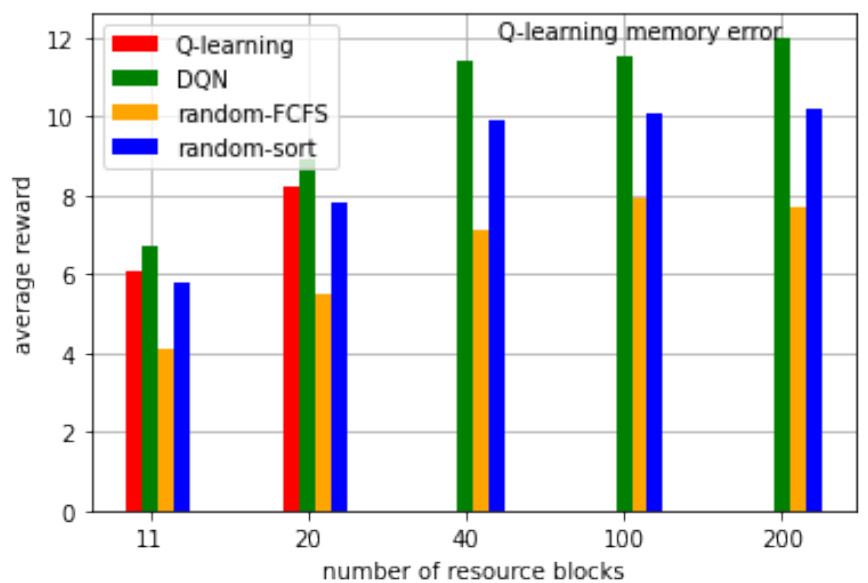

Fig. 8: The comparison of Q-learning, deep Q-learning (based on DQN) and random algorithms in terms of the average reward of an episode versus the number of RBs.

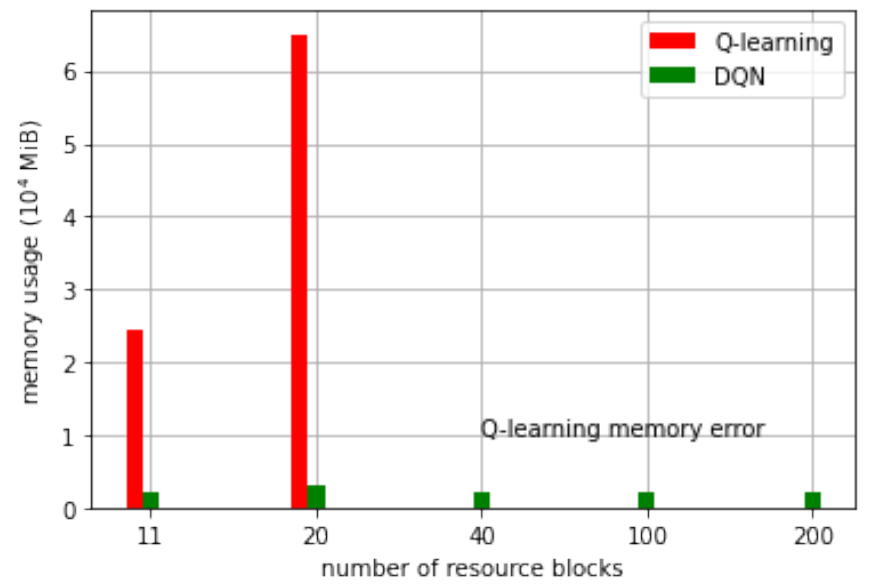

Fig. 9: The comparison of Q-learning and deep Q-learning (DQN) algorithms in terms of the memory usage of the algorithm versus the number of RBs.

learning when we vary $p_{I}$ for both (i.i.d. and session) arrival types of incumbent user. Q-learning adapts to the spectrum occupancy pattern of the incumbent user successfully and is effective in utilizing network resources that are left from the incumbent user's spectrum occupancy. As expected, the network utility achieved by Q-learning drops as $p_{I}$ increases but this drop is sub-linear with $p_{I}$.

The performance of Q-learning and deep Q-learning algorithms are compared in Figures 7, 8, and 9. Figure 7 shows the average reward of an episode versus the number of users. The performance of the Q-learning and deep Q-learning algorithms is close in terms of the average reward (less than $7 \%$ difference). Figure 8 shows the average reward of an episode versus the number of total available RBs. When the number of RBs are increased to more than or equal to 40 , a memory error is raised for Q-learning, due to large Q-table size not fitting in the memory. In the meantime, DQN scales up with the number of RBS and maintains the performance gain with respect to benchmarks. Figure 9 shows the occupied memory 


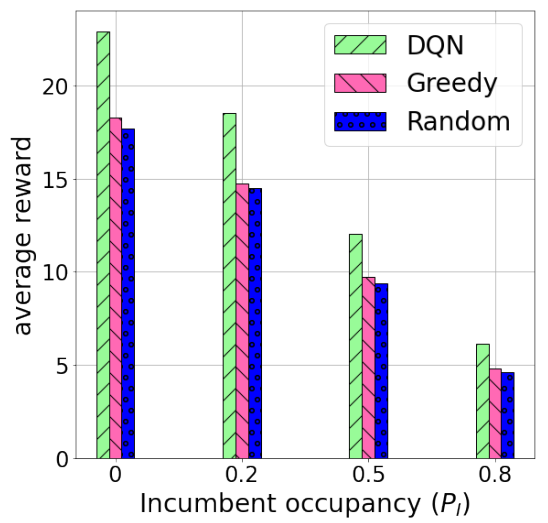

(a) Average reward versus incumbent occupancy probability $\left(p_{F}=0\right.$ and $p_{M}=$ $0)$. The available resources to requests decreases as the incumbent user occupies more resources.

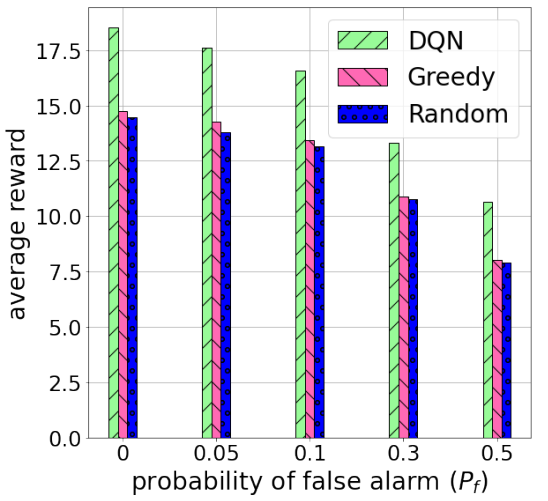

(b) Average reward versus probability of false alarm $\left(p_{I}=0.2\right.$ and $\left.p_{M}=0\right)$. The admission control should avoid what is thought to be an incumbent activity and the average reward decreases as a result.

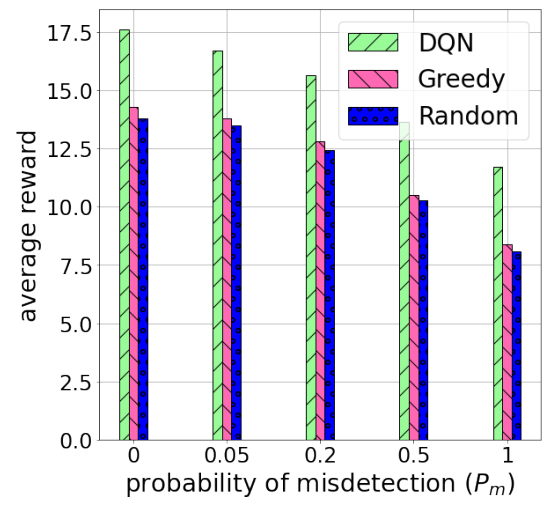

(c) Average reward versus probability of misdetection $\left(p_{I}=0.2\right.$ and $\left.p_{F}=0.05\right)$. As misdetection error increases, the inability to detect PU causes the average reward to decrease.

Fig. 10: Average reward of an episode versus the incumbent user activity and detection errors.

of different algorithms versus the number of total available RBs. Since the amount of occupied memory increases with the state-action space in the Q-learning algorithm, increasing the number of RBs leads to a memory error in case of using the Q-learning algorithm, while the occupied memory of the DQN algorithm remains almost the same.

We now have a closer look at the size of Q-table in Q-learning case. The Q-table has size $N_{S} \times N_{A}$, where $N_{S}$ is the number of states and $N_{A}$ is the number of actions. The action is either accepting a request or not, so $N_{A}=2$. The state includes information on the number of occupied RBs (from the set $\{0,1, \cdots, R\}$ ) by an incumbent user or by requests, amount of computational resources used (from the set $\{0,0.02, \cdots, 1\}$ ), amount of communication power used (from the set $\{0,1, \cdots, 5\}$ ), number of RBs required (from the set $\{1,2, \cdots, R\}$ ), communication power determined by throughput requirement (from the set $\{1,2, \cdots, 5\})$, the amount of processor usage required (from the set $\{0.02,0.04, \cdots, 1\}$ ), weight (from the set $\{1,2, \cdots, 5\}$ ), and duration of the considered request (from the set $\{1,2, \cdots, 10\}$ ). We set $R=11$ for most of the results. Thus, $N_{S}=12 \times 11 \times 5 \times 50 \times 5 \times 10=5.049 \times 10^{8}$. As this large Q-table size translates to a large memory requirement limiting practical implementation, deep Q-learning appears as the feasible solution.

Figure 10 shows the average reward of an episode versus the incumbent user activity $\left(p_{I}\right)$, probability of false alarm $\left(p_{F}\right)$, and probability of misdetection $\left(p_{M}\right)$. As shown in Figure 10a the average reward of an episode decreases as $p_{I}$ increases, due to less available resources. In Figure 10b, the average reward is shown to decrease with increasing $p_{F}$. The reason is that the admission control avoids the resources falsely thought to be occupied by an incumbent user. As shown in Figure 10c, as $p_{M}$ increases, the average reward of an episode decreases. This is due to failing in detection of the incumbent user activity, since at the end of each time slot the requests which had occupied overlapping RBs are considered failed and are sent back to the waiting queue. These results show that deep Q-learning for 5G RAN slicing adapts to spectrum dynamics in terms of incumbent behavior as well as sensing errors, and significantly improves the average reward with respect to the benchmark schemes. This adaptation is essential to enable operations for coexistence of $5 \mathrm{G}$ with the incumbent user such as in the CBRS band, ensuring that $5 \mathrm{G}$ causes minimal disruption to the incumbent user while seizing all the opportunities to occupy the shared spectrum. While the errors in spectrum sensing considered in Figure 10 certainly have an impact on efficient resource allocation, the results indicate that the use of ML for intelligent admission control can effectively improve resource allocation with respect to the baseline algorithms.

\section{CONCLUSION}

In this paper, we studied the problem of dynamic admission control for network slicing in 5G RAN and proposed a reinforcement learning-based solution that solves the underlying optimization problem over a time horizon. Our solution applies to the $5 \mathrm{G}$ spectrum coexistence scenarios, where $5 \mathrm{G}$ needs to share the spectrum with an incumbent user (subject to spectrum sensing errors). We considered both communication (frequency-time resource blocks and transmit powers) and computational (CPU usage) resources that are assigned to the incoming network slicing requests for downlink communications from the gNodeB to UEs. Each request comes with latency, rate, and CPU usage requirements and each request has a weight based on its priority. We constructed an reinforcement learning solution, where the state represents the available network resource information as well as request characteristics, actions are admitting/rejecting network slice requests and rewards are measured by the network utility, namely the weighted sum of satisfied network slicing requests. The underlying states that transition over time correspond 
to the available resources that become temporarily blocked for either granted network slices or due to the spectrum use of incumbent users. We presented Q-learning and deep Qlearning solutions to find the optimal admission control policy. We showed that these solutions outperform the baselines significantly in terms of the network utility, and deep Q-learning outperforms Q-learning in terms of the average reward, memory usage, and scalability. For the $5 \mathrm{G}$ spectrum coexistence scenarios, we showed that the reinforcement learning solutions can effectively allocate resources to network slices when some resources become unavailable randomly due to sharing the spectrum opportunistically with incumbent users, even in the presence of misdetection and false alarm errors for spectrum sensing.

\section{REFERENCES}

[1] Y. Shi, Y. E. Sagduyu, and T. Erpek, "Reinforcement learning for dynamic resource optimization in 5G radio access network slicing," in IEEE International Workshop on Computer Aided Modeling and Design of Communication Links and Networks (CAMAD), 2020.

[2] J. Koo, V. B. Mendiratta, M. R. Rahman, and A. Walid, "Deep reinforcement learning for network slicing with heterogeneous resource requirements and time varying traffic dynamics," in International Conference on Network and Service Management (CNSM), 2019, pp. 1-5.

[3] H. Halabian, "Distributed resource allocation optimization in 5G virtualized networks," IEEE Journal on Selected Areas in Communications, vol. 37, no. 3, pp. 627-642, 2019.

[4] S. D'Oro, F. Restuccia, and T. Melodia, "Toward operator-to-waveform 5G radio access network slicing," CoRR, vol. abs/1905.08130, 2019.

[5] A. Kaloxylos, "A survey and an analysis of network slicing in 5G networks," IEEE Communications Standards Magazine, vol. 2, no. 1, pp. 60-65, 2018.

[6] X. Foukas, G. Patounas, A. Elmokashfi, and M. K. Marina, "Network slicing in 5G: Survey and challenges," IEEE Communications Magazine, vol. 55, no. 5, pp. 94-100, 2017.

[7] J. Ordonez-Lucena, P. Ameigeiras, D. Lopez, J. J. Ramos-Munoz, J. Lorca, and J. Folgueira, "Network slicing for $5 \mathrm{G}$ with SDN/NFV: Concepts, architectures, and challenges," IEEE Communications Magazine, vol. 55, no. 5, pp. 80-87, 2017.

[8] P. Rost, C. Mannweiler, D. S. Michalopoulos, C. Sartori, V. Sciancalepore, N. Sastry, O. Holland, S. Tayade, B. Han, D. Bega, D. Aziz, and H. Bakker, "Network slicing to enable scalability and flexibility in 5G mobile networks," IEEE Communications Magazine, vol. 55, no. 5, pp. 72-79, 2017.

[9] Code of Federal Regulations, "Citizens Broadband Radio Service," Title 47, Part 96, 2015

[10] S. D'Oro, F. Restuccia, A. Talamonti, and T. Melodia, "The slice is served: Enforcing radio access network slicing in virtualized 5G systems," in IEEE Conference on Computer Communications (INFOCOM), 2019, pp. 442-450.

[11] V. Sciancalepore, K. Samdanis, X. Costa-Perez, D. Bega, M. Gramaglia and A. Banchs, "Mobile traffic forecasting for maximizing 5G network slicing resource utilization," in IEEE Conference on Computer Coтmunications (INFOCOM), 2017, pp. 1-9.

[12] A. NAKAO and P. DU, "Toward in-network deep machine learning for identifying mobile applications and enabling application specific network slicing," IEICE Transactions on Communications, vol. E101.B, 012018.

[13] A. Thantharate, R. Paropkari, V. Walunj, and C. Beard, "DeepSlice: A deep learning approach towards an efficient and reliable network slicing in 5G networks," in IEEE Annual Ubiquitous Computing, Electronics Mobile Communication Conference (UEMCON), 2019, pp. 0762-0767.

[14] R. Li, Z. Zhao, Q. Sun, C. I, C. Yang, X. Chen, M. Zhao, and H. Zhang, "Deep reinforcement learning for resource management in network slicing," IEEE Access, vol. 6, pp. 74 429-74 441, 2018.

[15] H. Wang, Y. Wu, G. Min, J. Xu, and P. Tang, "Data-driven dynamic resource scheduling for network slicing: A deep reinforcement learning approach,” Information Sciences, vol. 498, pp. 106-116, 2019.
[16] Q. Liu and T. Han, "When network slicing meets deep reinforcement learning," in International Conference on Emerging Networking EXperiments and Technologies, 2019, pp. 29--30.

[17] Z. Xu, Y. Wang, J. Tang, J. Wang, and M. C. Gursoy, "A deep reinforcement learning based framework for power-efficient resource allocation in cloud RANs," in IEEE International Conference on Communications (ICC), 2017, pp. 1-6.

[18] J. A. Ayala-Romero, A. Garcia-Saavedra, M. Gramaglia, X. CostaPerez, A. Banchs, and J. J. Alcaraz, "Vrain: A deep learning approach tailoring computing and radio resources in virtualized RANs," in Annиal International Conference on Mobile Computing and Networking, 2019.

[19] N. C. Luong, D. T. Hoang, S. Gong, D. Niyato, P. Wang, Y. Liang, and D. I. Kim, "Applications of deep reinforcement learning in communications and networking: A survey," IEEE Communications Surveys \& Tutorials, vol. 21, no. 4, pp. 3133-3174, 2019.

[20] H. Ye, G. Y. Li, and B. F. Juang, "Deep reinforcement learning based resource allocation for $\mathrm{V} 2 \mathrm{~V}$ communications," IEEE Transactions on Vehicular Technology, vol. 68, no. 4, pp. 3163-3173, 2019.

[21] N. Abuzainab, T. Erpek, K. Davaslioglu, Y. E. Sagduyu, Y. Shi, S. J. Mackey, M. Patel, F. Panettieri, M. A. Qureshi, V. Isler, and A. Yener, "Qos and jamming-aware wireless networking using deep reinforcement learning," in IEEE Military Communications Conference (MILCOM), 2019, pp. 610-615.

[22] G. Sun, Z. T. Gebrekidan, G. O. Boateng, D. Ayepah-Mensah, and W. Jiang, "Dynamic reservation and deep reinforcement learning based autonomous resource slicing for virtualized radio access networks," IEEE Access, vol. 7, pp. 45 758-45 772, 2019.

[23] Y. Shi, Y. E. Sagduyu, T. Erpek, and M. C. Gursoy, "How to attack and defend 5G radio access network slicing with reinforcement learning," arXiv preprint arXiv:2101.05768, 2021.

[24] Y. Shi and Y. E. Sagduyu, "Adversarial machine learning for flooding attacks on 5G radio access network slicing," in IEEE International Conference on Communications Workshops (ICC Workshops), 2021.

[25] 3GPP TS 38.306:, "NR; User Equipment (UE) radio access capabilities."

[26] 3GPP TS 38.211:, "NR; Physical channels and modulation."

[27] D. Bega, M. Gramaglia, M. Fiore, A. Banchs, and X. Costa-Perez, "Deepcog: Cognitive network management in sliced 5G networks with deep learning," in IEEE Conference on Computer Communications (INFOCOM), 2019, pp. 280-288.

[28] R. Caromi, M. Souryal, and W. Yang, "Detection of incumbent radar in the 3.5 GHZ CBRS band," in IEEE Global Conference on Signal and Information Processing (GlobalSIP), 2018, pp. 241-245.

[29] W. M. Lees, A. Wunderlich, P. J. Jeavons, P. D. Hale, and M. R. Souryal, "Deep learning classification of $3.5-\mathrm{GHz}$ band spectrograms with applications to spectrum sensing," IEEE Transactions on Cognitive Communications and Networking, vol. 5, no. 2, pp. 224-236, 2019.

[30] V. Mnih, K. Kavukcuoglu, D. Silver, A. A. Rusu, J. Veness, M. G. Bellemare, A. Graves, M. Riedmiller, A. K. Fidjeland, G. Ostrovski et al., "Human-level control through deep reinforcement learning," Nature, vol. 518, no. 7540, pp. 529-533, 2015. 